\title{
Effect of dietary carob bean gum and live yeast in the prevention of Escherichia coli in weaning pigs
}

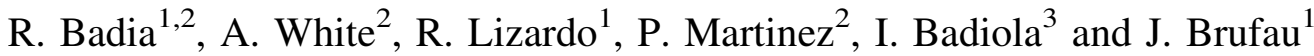 \\ ${ }^{1}$ IRTA - Animal Nutrition, Spain, ${ }^{2}$ IBB - Immunologia, UAB, Spain and ${ }^{3}$ CReSA, Spain
}

Gram-negative bacteria adhere to mannose rich substrates. Carob bean gum (CBG) and live yeast Saccharomyces cerevisae are natural mannose rich products. Moreover, live yeast has beneficial effects in the prevention and treatment of intestinal disorders. The aim was to study the inclusion of CBG (Salmosan ${ }^{\mathrm{TM}}$, ITPSA, Spain) or live yeast (S. cerevisae SC47, Biosaf ${ }^{\mathrm{TM}}$, Lesaffre, France) in diets for weaned pigs challenged or not with Escherichia coli K99 and to evaluate their role as immunological enhancers of the intestinal barrier.

A total number of 25 Landrace*Duroc 4 weeks weaned pigs were used in this trial. There was a control non-infected group and four groups challenged with E. coli: control infected animals and infected animals supplemented with Colistin, CBG or live yeast. Pigs were fed for 2 weeks with experimental diets before the challenge. Animals were orally challenged with $1 \times 10^{8}$ cfu of E. coli K99. Pigs were euthanized 3 days after the challenge. Blood, bile, ileum and mesenteric lymph node (MLN) were sampled.

Our results showed an increase of CRP levels in the control challenged compared to the control group. Groups challenged by E. coli and fed with CBG and live yeast, had similar CRP levels compared to the Colistin and control group. Total sIgA was higher in all infected groups than in control pigs. TLR2 and TLR4 are involved in the recognition of bacteria. In ileum TLR4 was upregulated in control $+E$. coli compared with control pigs. In MLN, both receptors were upregulated in control $+E$. coli compared with control pigs. Control group and pigs supplemented with live yeast, showed similar expression of TLR2 and TLR4 in both tissues. The infection was reduced in pigs fed with live yeast probably to an exclusion effect avoiding the adhesion of $E$. coli to the intestinal mucosa.

It can be concluded that carob bean gum and live yeast $S$. cerevisae may reduce the risk of $E$. coli infection in pigs. Both products seem to be suitable as alternative to dietary antibiotics. 